\title{
DISCOURS POUR LE DOCTORAT HONORIS CAUSA EN PÉDAGOGIE
}

Por Florence Gillet, tradução para o francês do original LUBICH, Chiara Lezione per laurea Honoris Causa in Pedagogia.

\author{
Votre Éminence, Monsieur le cardinal James Hickey, \\ Monsieur le Président O’Connell \\ Excellence, Mgr Montalvo, \\ Vos Excellences, \\ Mesdames et Messieurs, membres du Conseil d'Administration, \\ Mesdames et Messieurs les Professeurs et membres du Sénat académique, \\ Messieurs les Ministres, et vous qui représentez les autorités civiles, \\ Mesdames et Messieurs les Professeurs, \\ Mesdames et Messieurs, chers amis,
}

Je remercie cordialement cette illustre Université d'avoir bien voulu me remettre ce doctorat en Pédagogie et le remettre, à travers moi, au mouvement des Focolari. Sans doute s'agit-il de la reconnaissance de la contribution que nous avons peut-être apportée à la formation de la personne et de la société dans le domaine de l'éducation.

Le mobile de nos actions est religieux et se fonde toujours - y compris dans le domaine éducatif - sur le Christ qui est allé jusqu'à référer à sa personne le concept d'éducateur: “ Ne vous faites pas donner le titre de Rabbi car vous n'avez qu'un seul maître, le Christ" (Mt 23,10).

Avant de traiter l'aspect de l'éducation dans notre Mouvement, qui a également pour nom “OEuvre de Marie", je pense qu'il est bon, au moins pour ceux qui ne le connaîtraient pas, de présenter la spiritualité qui l'anime et qui a suscité un nouveau style de vie.

L'OEuvre de Marie est un mouvement d'Église. Sa diffusion (en 200 pays) et le nombre de ses adhérents (plusieurs millions) prouvent qu'il s'agit d'une OEuvre de Dieu. C'est ce que soulignent Jean-Paul II et l'Église.

L'objectif du mouvement est d'apporter sa contribution à la réalisation du Testament de Jésus : "Que tous soient un " (Jn 17,21). Le moyen de l'atteindre est sa spiritualité, qui est à la fois personnelle et communautaire. 
Les grands axes constitutifs de cette spiritualité s'appuient sur des paroles ou des idées évangéliques qui en constituent les piliers. On peut les comprendre dans toute leur dimension en observant par quelle stratégie l'Esprit Saint les a gravées profondément dans nos coeurs aux tout débuts de notre nouvelle vie.

Remontons aux années quarante, en Italie, pendant la seconde guerre mondiale.

Un jour, alors que j'accomplis un acte d'amour, je ressens que Dieu m'appelle à me donner entièrement à Lui et je le fais le 7 décembre 1943, qui est considéré depuis lors la date de naissance du mouvement.

Pour différentes raisons, j'entre en contact avec des jeunes filles de mon âge qui veulent suivre ma voie.

Le 13 mai 1944 Trente, ma ville natale, est victime, une fois encore, d'un terrible bombardement.

Avec mes compagnes, nous nous sommes réfugiées un jour dans une cave obscure et, à la lumière d'une bougie, nous lisons l'évangile. Je tombe sur la prière de Jésus avant de mourir : "Père... que tous soient un " (cf. Jn 17,21). Ces paroles nous frappent au point que nous sommes intimement convaincues que c'est pour cette page-là de l'Évangile que nous sommes nées.

Les bombardements continuent et avec eux disparaissent les personnes ou les choses qui constituent nos idéaux de jeunes : le désir de fonder une famille, puisque le fiancé ne revient pas du front; poursuivre nos études devient une chimère ainsi que d'avoir une maison, et ainsi de suite.

La leçon que Dieu nous donne à travers ces circonstances est claire : “ Tout est vanité des vanités." (Qo 1,2). Tout passe.

Alors, une question se fait jour en moi : “Existe-t-il un but, un idéal pour lequel il vaille la peine de vivre, qu'aucune bombe ne puisse détruire? ".

Et je perçois tout de suite la réponse : "Oui, cet idéal existe : c'est Dieu ”.

Avec mes compagnes, nous décidons de faire de Dieu notre raison de vivre.

Mais qui est Dieu?

Dès que ce nom est apparu à l'horizon de nos vies, nous avons compris ou, mieux, redécouvert avec une clarté éblouissante que Dieu est Amour. À partir de ce moment-là nous nous sommes senties enveloppées de son amour. 
Nous connaissions déjà son existence, toutefois pour nous Dieu restait lointain, au-delà du firmament, inaccessible.

D’un seul coup tout changeait. Comme si nos yeux s'étaient ouverts, nous découvrions que Dieu, parce qu'il est Amour, était proche de nous, qu'il nous suivait à chaque pas, qu'il se trouvait derrière les circonstances joyeuses ou tristes de notre vie, qu'il savait tout de nous.

Croire à Dieu-amour: telle fut la première idée-force de cette nouvelle spiritualité. Mais si Dieu est amour — pensions-nous — comment nous comporter à son égard?

Jésus, par sa vie, nous indiquait la réponse : il avait aimé le Père en faisant sa volonté. Il devait en être de même pour nous.

Faire la volonté de Dieu est la deuxième idée-force de notre spiritualité.

Les bombes, qui tombaient jour et nuit, nous obligeaient à nous réfugier dans les abris, parfois onze fois par jour. Nous ne pouvions rien emporter avec nous, à l'exception d'un petit livre : l'Évangile. Et nous étions sûres que nous y aurions découvert la volonté de Jésus et ses commandements

Nous l'ouvrions et les paroles que nous y trouvions s'allumaient d'une clarté très vive. Nous les comprenions comme si nous ne les avions jamais entendues auparavant et une force qui venait, pensons-nous, de l'Esprit Saint, nous poussait à les mettre en pratique.

Nous lisions : "Aime ton prochain comme toi-même" (Mt 19,19). Mais où est le prochain?

Il était là, en toutes ces personnes durement frappées par la guerre et qui souffraient de la faim, de la soif, étaient blessées, sans vêtements, sans toit. Et, sans attendre, nous allions prendre soin d'elles.

L'Évangile nous promettait : “Demandez et vous recevrez” (Mt 7,7 ; Lc 11,9).

Nous demandions pour les pauvres et à chaque fois nous recevions toute sorte de choses que nous portions à ceux qui en avaient besoin.

Et, à ce point, je dois m'arrêter de suivre mon texte pour raconter un épisode que quelqu'un ici présent m'a demandé. Nous étions donc au début de notre expérience, quand un pauvre vient me demander de lui donner une paire de chaussures pointure 42. Or, en pleine guerre où les chaussures étaient une denrée introuvable, trouver la pointure 42 était impossible. Je me souviens que je suis allée à l'église devant le tabernacle et, consciente que Jésus est présent dans les pauvres, je lui ai demandé : “ Jésus donne-moi une paire de chaussures $n^{\circ} 42$ pour toi dans ce pauvre ". Aussitôt sortie de l'église, une jeune femme s'approche de moi et me tend un paquet " pour tes pauvres", dit-elle. Il contenait une paire de chaussures pointure 42 . 
“Donnez et vous recevrez" (Lc 6,38), avons-nous lu. Nous donnons. Un jour, il y avait une seule pomme à la maison que nous avons donnée au pauvre qui est venu frapper à notre porte. Dans la matinée, il en est arrivé un sac, que nous avons encore donné. Le soir il en est arrivé une pleine valise.

Jésus avait promis et maintenait ses promesses. L'Évangile était donc vrai.

Cette constatation nous donnait des ailes pour poursuivre le chemin sur lequel nous venions de nous engager. Nous racontions cela autour de nous et, en nous rencontrant, les gens avaient l'impression de rencontrer Jésus vivant.

Mettre en pratique la Parole de Dieu est le troisième point de notre spiritualité.

Toutes les paroles de Jésus nous interpellaient. Mais bien vite l'Esprit Saint a mis en relief celles qui concernent l'amour, l'amour évangélique.

Et vivre l'amour est devenu le quatrième pilier de notre spiritualité.

Cependant, comme nous étions sans cesse exposées à la mort, un jour nous nous sommes demandé : “Existe-t-il une volonté de Dieu qui tienne particulièrement à coeur à Jésus ? Car c'est celle-là que nous aimerions accomplir avant de mourir ".

Il y avait dans l'évangile un commandement que Jésus avait défini " sien " et “ nouveau ". Voilà exactement ce que nous cherchions : "Je vous donne un commandement nouveau : aimez-vous les uns les autres comme je vous ai aimés. Il n'y a pas de plus grand amour que de donner sa vie pour ses amis " (cf. Jn 15,12-13).

Nous voulons nous mettre à le vivre et nous le scellons par un pacte. Nous nous regardons dans les yeux et nous disons les unes aux autres : "Je suis prête à mourir pour toi. Moi pour toi. Toutes pour chacune."

À partir de ce moment-là, notre vie a changé du tout au tout.

Que s'était-il passé ? En posant cet acte, le feu de la charité réciproque s'était mis à brûler parmi nous. Et l'amour nous unissait désormais, au point de réaliser ce que Jésus avait souhaité lorsqu'il avait dit : “Là où deux ou trois sont réunis en mon nom (en mon amour) je suis au milieu d'eux" (Mt 18,20). Il était donc venu établir sa demeure au milieu de nous. Et ce que nous éprouvions en nous-mêmes de si nouveau, de si beau, de si lumineux était l'effet de sa présence.

L'amour réciproque et Jésus au milieu de nous : autres points de notre spiritualité.

Évidemment, nous n’arrivions pas toujours à vivre dans cette dimension-là. Il suffisait parfois d'un défaut, même petit, pour offusquer la beauté de cette vie d'unité.

L'Évangile, là encore, allait nous apprendre comment affronter cela. 
Par une circonstance fortuite, nous avions découvert que la souffrance de Jésus avait atteint son paroxysme lorsque, sur la croix, il était allé jusqu'à expérimenter l'abandon du Père et s'était écrié : «Mon Dieu, mon Dieu, pourquoi m’as-tu abandonné ?» (Mc 15,34 ; Mt 27,46).

Touchées profondément, nous avons pris la décision de suivre désormais Jésus dans son abandon.

À partir de ce moment-là, nous avons découvert son visage partout et où qu'il soit nous l'avons accueilli avec amour : dans nos souffrances personnelles, chez les personnes seules comme lui, abandonnées comme lui, affligées, dans les divisions du monde.

Jésus crucifié et abandonné : un autre point très important de notre spiritualité.

Mais Jésus n'avait-il pas dit : “A ceci tous vous reconnaîtront pour mes disciples : à l'amour que vous aurez les uns pour les autres. " (Jn 13,35) ? et ailleurs, n'avait-il pas dit : "Qu'ils soient un... afin que le monde croie " $($ Jn 17, 21) ?

Voilà que la foi des gens autour de nous était ravivée, d'autres personnes la retrouvaient, d'autres encore croyaient en Jésus pour la première fois.

L'amour réciproque, qui engendre la présence de Jésus au milieu de nous, nous rendait aptes à réaliser l'unité pour laquelle Jésus a prié : “Qu'ils soient un comme toi et moi " (Jn 17,21).

L'unité : autre point de notre spiritualité.

Voilà un rapide tour d'horizon de notre OEuvre, présentée sous son aspect spirituel.

Mais on peut considérer aussi notre mouvement d'un point de vue théologique, philosophique, culturel, social, économique, artistique et éducatif, ainsi que d'un point de vue oecuménique, interreligieux.

Je vais donc maintenant chercher à exposer quelques-unes des répercussions des principales idées-forces de cette spiritualité dans le domaine de la pédagogie.

On peut considérer notre Mouvement et les étapes de son histoire comme un événement éducatif important, assez extraordinaire. On y retrouve en effet tous les éléments qui constituent l'éducation et on peut en tirer également une théorie de l'éducation ainsi qu'une pédagogie bien précise qui fonde notre praxis éducative.

Mais demandons-nous d'abord : qu'est-ce que l'éducation?

On peut la définir ainsi : c'est l'itinéraire que parcourt l'éduqué (individu ou communauté) avec l'aide de l'éducateur (ou de plusieurs éducateurs) dans la perspective 
de parvenir à réaliser ce qu'il devrait devenir, d'atteindre un but que l'on considère valable pour l'homme et l'humanité.

Quels sont les éléments caractéristiques de notre pédagogie qui sont liés aux points principaux de la spiritualité que nous vivons?

Si nous prenons le premier point : la révélation - permettez-moi cette expression - de Dieu comme Amour, nous constatons que notre histoire est marquée dès ses débuts par la présence d'un unique éducateur, l'Éducateur par excellence : Dieu Amour, justement, Dieu Père. C'est lui qui a pris l'initiative de nous éduquer, qui nous a accompagnés, qui nous a transformés, engendrés à une vie nouvelle - grâce à cette intentionnalité (ce projet clair, ndt) qui guide tout éducateur authentique - et cela, tout au long d'un immense itinéraire de formation personnelle et communautaire.

C'est Lui qui nous a permis - à nous et à beaucoup - de retrouver le sens de la Paternité au degré le plus élevé. Cette découverte a une portée incommensurable dans le monde d'aujourd'hui où une certaine culture tente d'affirmer - théoriquement et pratiquement - la mort de Dieu. L'éclipse du Père a entraîné l'éclipse de la figure paternelle, la perte de l'autorité dans les relations humaines et éducatives, un relativisme moral, une absence de normes de vie pour l'individu, ainsi que dans les relations interpersonnelles et sociales. Tout cela entraîne souvent de graves conséquences, comme les différentes formes de violence etc. et semble donner raison à Dostoïevski qui affirmait : "Tuer Dieu est le suicide le plus terrible " et encore : Si Dieu n'existe pas, alors tout est permis."

Nous avons eu la grâce de connaître Dieu. Et Dieu est amour. Loin d'être un juge lointain, un ennemi qui jalouse l'homme et l'écrase de sa puissance ou qui ne se soucie pas de Lui, c'est, au contraire, un éducateur qui reconnaît l'homme dans son identité personnelle, son unicité et qui l'exalte. Il aime l'homme et pour cette raison il est exigeant : en véritable éducateur il place haut la barre et éduque à la responsabilité, à l'engagement. Dieu est amour, et pour cette raison il nous a libérés du plus grand esclavage et nous a ouvert les portes de sa Demeure ; et nous savons à quel prix son Fils a payé ce rachat. Aucun éducateur n'a jamais eu une aussi grande considération de l'homme que Dieu, qui est mort pour lui. Dieu Amour a élevé l'homme, tout homme, à la très haute dignité de Fils et d'héritier. Tout homme !

C'est d'ailleurs à partir de cette constatation - nous sommes tous fils du même Père - que se fonde l'idée-force de Comenius ${ }^{1}$, illustre représentant de la pédagogie moderne : "Enseigner tout à tout le monde."

Et nous arrivons à un deuxième point fort de notre spiritualité : la Parole de Dieu.

1 Né en Moravie (Tchéquie) en 1592, mort en 1670, il a été le premier à tenter, à côté d'une première réforme pratique radicale, la première organisation systématique de la pédagogie comme science 
“Enseigner tout à tout le monde ", avons-nous dit, mais pour ce faire il est nécessaire de procéder par étapes, comme le notait Comenius lui-même. À bien y regarder, ce cheminement progressif nous a été enseigné par le Père lui-même lorsque, dès les tout premiers temps, il nous a suggéré, pour vivre sa Parole, de choisir une phrase de l'Évangile à la fois et de la mettre en pratique dans la vie de tous les jours, pendant un mois.

Cela nous a mis immédiatement en contact avec le “Tout”, puisqu'en chaque Parole Jésus est présent tout entier (et dans la Parole vécue c'est Lui qui vit en nous); en même temps, comme des enfants nourris de sa Parole, nous l'assimilions toujours davantage, grandissant jusqu'à devenir adultes dans la foi et dans la vie.

Grâce à ce simple moyen pédagogique, qui permet d'avancer graduellement tout en étant dans la plénitude, la lumière de notre Idéal s'est répandue et ne cesse de se répandre bien au-delà de notre mouvement en tant que forte expérience spirituelle et éducative qui ne cesse de s'étendre.

La caractéristique de la Parole de Dieu est d'être une Parole de Vie qui se traduit dans la vie concrète, dans un monde souvent empreint de verbalisme - et le domaine pédagogique n'y fait pas exception -.

Et nous avons touché du doigt la force éducative de cette parole toujours nouvelle et toujours vivante qui propose une autre façon de vivre et une remise en question de soi. $\mathrm{Au}$ fur et à mesure que nous en avons pétri notre vie, la Parole lui a conféré son unité existentielle, ce qui est la tâche spécifique et immense de l'éducation. La Parole aide à surmonter l'impression de fragmentation, de morcellement que l'homme ressent si souvent dans sa relation à lui-même, aux autres, à la société et à Dieu. Et elle fait ressortir en même temps le caractère propre, unique et original de chacun.

C'est cette unité existentielle entre Parole et Vie, entre dire et faire, qui, aux yeux de beaucoup, rend notre expérience crédible et convaincante. Cela provoque de profonds changements chez beaucoup et déclenche un authentique processus éducatif.

Un autre point : La volonté de Dieu.

En étant fidèles à la Parole de Dieu, nous avons peu à peu perdu notre «mauvaise» volonté " - celle qui nous tient encore prisonniers de notre moi égocentrique - et nous avons accompli la volonté de Dieu, qui nous porte à nous transcender nous-mêmes, à nous dépasser vers un Tu qui nous comble et nous libère.

Généralement par l'éducation morale, la personne passe progressivement de la phase initiale obligatoire de dépendance (moralité hétéronome) (c'est-à-dire où la moralité vient de l'extérieur, ndt) à une moralité autonome (c'est-à-dire où la moralité est intériorisée, ndt) (qui devrait caractériser l'adulte et le sujet mûr). Dans notre expérience éducative aussi, 
nous percevons qu'il y a un passage d'une adhésion initiale à la volonté d'un autre et à sa loi (qui se manifeste sous plusieurs formes) - à laquelle on tient comme un enfant qui s'abandonne totalement à la guide de l'adulte - à l'intériorisation de la Loi ; cela advient lorsque nous sentons que la loi est devenue notre loi et que nous en sommes pétris au point de nous sentir adultes, c'est-à-dire en mesure de dire : “Ce n'est plus moi qui vis, c'est le Christ qui vit en moi." (Gal 2,20).

Il y a ensuite le point de Jésus qui crie «Mon Dieu, mon Dieu, pourquoi m’as-tu abandonné ?» (Mc 15,34; Mt 27,46)

Jésus abandonné est la clé de notre vie, son secret, y compris dans le domaine de l'éducation. Il nous indique que l'horizon de notre action pédagogique est illimité, quelle est sa mesure et l'intensité qu'elle doit revêtir.

Qui donc est Jésus abandonné pour qui nous avons décidé d'avoir un " amour de prédilection" ? Il est l'image même de l'ignorant : il demande " pourquoi ?"; son ignorance est la plus tragique qui soit, sa question est la plus dramatique qui soit. Il est l'image même de la personne indigente, inadaptée, handicapée, de celui qui n'est pas aimé, du laissé-pour-compte, du marginal, et de toutes ces personnes dont la situation humaine et sociale requiert avec urgence une éducation appropriée. Jésus abandonné est le paradigme de celui qui, manquant de tout, a besoin de quelqu'un qui lui donne tout et qui entreprenne tout pour lui. Il est donc aussi le concept extrême, le paramètre, la référence, de l'éduqué qui en appelle à la responsabilité de l'éducateur. Il indique donc la mesure - une mesure sans bornes - d'un tel besoin d'éducation et, en même temps, la mesure - elle aussi sans bornes - de notre responsabilité de venir en aide et d'éduquer.

Jésus abandonné, toutefois, a surmonté son infinie douleur quand il a dit : "Entre tes mains, Père, je remets mon esprit" (Lc 23,46). Il nous enseigne donc également à considérer les difficultés, les obstacles, les épreuves, l'effort, l'erreur, l'échec, la souffrance, comme quelque chose à affronter, à aimer, à surmonter. Généralement nous les hommes, en quelque domaine que ce soit, nous cherchons par tous les moyens à éviter ce genre d'expériences. Dans le domaine de l'éducation aussi, on cherche le plus possible à préserver ceux qui nous sont confiés et à leur éviter les difficultés, on les surprotège, parfois par toute sorte de moyens. On leur présente ainsi une image de la vie confortable, comme celle d'une route facile, où les difficultés sont aplanies. Ce faisant, on ne leur donne pas l'outil qui leur permettrait de faire face aux inévitables épreuves de la vie et, en particulier, on les rend passifs et réfractaires par rapport aux responsabilités que chacun doit assumer par rapport à soi-même, aux autres, à la société.

Selon nous, en revanche, chaque difficulté doit être aimée et affrontée, justement en raison de notre choix de Jésus abandonné. L'éducation à affronter la difficulté, engagement 
qui concerne aussi bien l'éduqué que l'éducateur, est donc un autre point clé de notre pédagogie.

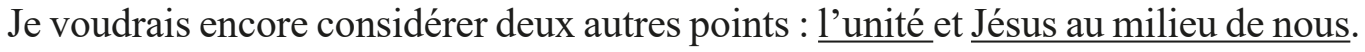

Au préalable, il faut répondre à cette question : quel est l'objectif de ce processus éducatif?

Notre objectif coïncide avec ce que nous pourrions définir comme étant l'objectif éducatif de Jésus : “Que tous soient un ". L'unité donc, unité profonde et ressentie avec Dieu et entre les hommes.

L'unité est une aspiration on ne peut plus actuelle. Malgré les multiples tensions du monde contemporain, notre planète, d'une façon qui peut paraître paradoxale, tend vers l'unité : l'unité est un signe des temps, une exigence de notre époque.

Cependant cet élan doit pouvoir émerger de façon positive - comme dans l'action éducative, du latin ex-ducere, ce qui mène en dehors de -. Cela implique que l'action éducative soit, à tous les niveaux, en cohérence avec les exigences de l'unité, et ait comme horizon l'édification de notre monde non pas comme une Babel sans âme, mais comme une expérience d'Emmaüs, de Dieu avec nous, qui embrasse l'humanité entière. Cela peut sembler un projet utopique, mais tout projet pédagogique authentique présente une part d'utopie qui doit être comprise comme la force qui nous pousse à édifier ici-bas un monde qui n'existe pas encore, mais qui devrait exister. L'éducation, dans cette perspective, est considérée comme un moyen pour atteindre ce but utopique.

Dans notre pédagogie où le plan spirituel et le plan humain sont étroitement liés et s'unifient (en raison de l'incarnation), l'Utopie n'est ni un rêve, ni une illusion, ni un but inaccessible. Elle est parmi nous et nous en constatons les fruits quand nous réalisons les paroles de Jésus : “Là où deux ou trois sont réunis en mon nom, je suis au milieu d'eux ” (cf. Mt 18,20). La finalité, le but le plus élevé, devient alors une réalité.

Nous expérimentons ici la plénitude de la vie que Jésus nous a donnée, la vie de Dieu, la relation trinitaire, la socialité la plus authentique, où se réalise une synthèse merveilleuse entre les deux buts de l'éducation : former l'individu et construire la communauté. Nous pensons que notre expérience de spiritualité communautaire trinitaire réalise pleinement les idées soutenues par d'illustres penseurs de l'histoire de la pédagogie. Bien que leurs prémisses aient été souvent différentes des nôtres, ils ont insisté sur l'importance de l'éducation pour édifier une société fondée sur des relations authentiquement démocratiques. Nous pensons ici, pour ne citer qu'un nom, à l'apport précieux que les États-Unis ont donné à la pédagogie en la personne de John Dewey. Dans son oeuvre nous trouvons beaucoup d'affinités avec la récente "pédagogie de communauté " qui proclame la nécessité de conjuguer le fait de promouvoir la personne et celui de promouvoir la communauté. 
Bien entendu, c'est sur l'invitation de Jésus : “Aimez-vous comme je vous ai aimés... Soyez une seule chose... ", et donc sur une motivation de nature religieuse que se fonde notre vie communautaire. Toutefois ses conséquences sur le plan éducatif sont tout à fait remarquables. La finalité de l'éducation, reconnue depuis toujours (former l'homme, l'amener à acquérir son autonomie) se réalise, paradoxalement, dans la formation de l'homme-relation, (l'homme en relation) ce qui signifie pour nous l'homme icône de la Trinité, capable d'auto-transcendence continuelle dans la réalité de Jésus au milieu de nous. C'est par cette praxis, cet exercice spirituel et éducatif de l'amour réciproque, de se consumer en un (exercice que font tous les membres du mouvement, tous appelés à vivre une expérience communautaire dans des petits groupes) que nous oeuvrons à la réalisation de l'objectif par excellence, celui qui se résume dans la prière-testament de Jésus : " Que tous soient un ". En tant qu'instruments guidés par Lui, c'est pour cette Utopie qui ne cesse de devenir réalité que nous voulons consacrer notre vie.

Et c'est à travers une sérieuse éducation que nous pouvons acquérir la capacité, en tant qu'individus et en tant que communauté, de collaborer, de dialoguer, de rencontrer d'autres personnes, d'autres mouvements, etc. Enfin, c'est à travers une sérieuse éducation que nous pouvons viser - avec la grâce de Dieu - à atteindre la sainteté, personnelle et communautaire.

Marie est notre modèle car elle a vécu de façon sublime les points pédagogiques que je viens d'énoncer.

Naturellement notre modèle est aussi Jésus, qui a su accomplir cet itinéraire pédagogique, ce va-et-vient entre l'abandon et la Trinité et qui, au cours de son expérience terrestre, a vécu avec une intensité unique ses relations interpersonnelles, en pratiquant l'empathie, l'acceptation, l'espérance, le combat éducatif, la vie d'unité avec son Père et avec les siens : il est le modèle le plus authentique et le plus exigeant de tout éducateur.

Chers amis,

J'espère que ce rapide exposé a mis suffisamment en évidence l'expérience pédagogique qui ressort de la vie de notre mouvement, et qu'il peut vous faire comprendre quel grand honneur représente pour moi cette remise de doctorat en pédagogie.

Merci de votre écoute. Que Jésus Maître fasse de nous tous des éducateurs authentiques et de valeur. 